EESTI NSV TEADUSTE AKADEEMIA TOIMETISED. X KÖDE

FOOSIKALIS-MATEMAATILISTE JA TEHNILISTE TEADUSTE SEERIA, 1961, NR. 4

ИЗВЕСТИЯ АҚАДЕМИИ НАУК ЭСТОНСКОИ ССР. ТОМ Х

СЕРИЯ ФИЗИКО-МАТЕМАТИЧЕСКИХ И ТЕХНИЧЕСКИХ НАУК, 1961, ㅅ́ 4

\title{
ЕЩЕ РАЗ О ФОРМУЛАХ А. ВАШИЧЕКА
}

\author{
П. КАРД, \\ член-корреспондент Академии наук Эстонской ССР
}

Профессор А. Вашичек опубликовал в прошлом году в этом журнале ответ [1] на критическую статью автора [2], написанную по поводу пересмотра Вашичеком теории отражения и пропускания света поглощающими интерференционными покрытиями. В этой статье Вашичек пытается найти новое обоснование для своих формул, однако, по мнению автора, его доводы неубедительны. В настоящей работе покажем, вопервых, что ответ Вашичека ни в коей мере не отводит, и не ослабляет критику, и. во-вторых, что его новые соображения тоже ошибочны и не вносят в рассматриваемую проблему ничего существенно нового. Попутно будет дана новая, нанболес общая формулировка оптического принщипа обратимости.

\section{1. Теория Максвелла и формулы Вашичека}

Обычная теория интерференции света в тонкослойных покрытиях основана на общепринятой теории электромагнитного поля Максвелла. Сюда относится и случай отраження и пропускания света тонким металлическим слоем. Формулы, выведенные для этого случая Мурманом [3], получены им непосредственно на основе этой теории.

Формулы Вашичека, однако, не согласуются с формулами Мурмана; кроме того, Вашичек получает свои формулы не на основании теории Максвелла, а из ряда побочных соображений. Во всяком случае, мы были бы вправе пожелать, чтобы Вашичек либо доказал, что формулы Мурмана противоречат теории Максвелла, либо, что его собственные формулы не противоречат этой теории. В статье же Вашичека [1'] мы не находим ни того, ни другого; его заявление о том, что «... ревизия не касается основ теории Максвелла», не получает подтверждения. Вместо этого в статье говорится о двух принципах обратимости, но не дается никаких пояснений относительно связи этих принципов с теорией Максвелла.

\section{2. Энергетическая проверка}

Вашичек утверждает, что фазы в формулах Мурмана неверны и что это было доказано с помощью энергетической проверки. Однако, автор настоящей статьи достаточно подробно показал (см. $\left.\left[{ }^{2}\right]\right)$, что это не так. Формулы Мурмана полностью выдерживают энергетическую проверку. Ведь они правильно выведены из теории Максвелла, в которой закон сохранения энергии выполняется автоматически. Предложенные же Вашичеком новые формулы оказываются, следовательно, лишенными достаточного основания. 


\section{3. Принцип обратимости}

Вашичек вводит в рассмотрение два принципа обратимости, которые он связывает с именами Власова и Стокса. Однако, как будет показано ниже. Вашпчек делает из принципа Стокса ошибочные выводы. Исправление же его ошибки вновь дает обычную теорию, включая формулы Мурмана.

В сущности, двух принципов обратимости нет, а есть только один принцип. То, что Вашичек называет принципом обратимости Власова, есть лишь частный случай более общего принципа, и из этого частного принципа Вашичек делает неправомерные выводы. Так, он полагает, что формулы Мурмана вытекают из принципа Власова. На самом же деле, как будет показано ниже, если выводить формулы Мурмана с помощью принципа обратимости, то для этого следует воспользоваться обобщенным принципом.

С Вашичеком можно согласиться лишь в том, что необходимо применять единый принцип обратимости. Но как его сформулировать? Очевидно, единый принцип обратимости должен вытекать из системы уравнений Максвелла. В частном случае, когда поглощающие среды отсутствуют, принцип обратимости хорошо известен; его можно найти, например, в цитируемой Вашичеком книге А. А. Власова [ $\left.{ }^{4}\right]$. Едва ли правильно, однако, называть его, как это делает Вашичек, принципом Власова, так как он был известен и ранее. Состоит он в том, что в случае отсутствия поглощающих (проводящих) сред уравнения электродинамики Максвелла не изменяются при инверсии времени, $t \rightarrow-t$, если заменить $\mathbf{E} \rightarrow \mathbf{E}, \mathbf{H} \rightarrow-\mathbf{H}, \mathbf{Q} \rightarrow \underline{\text {. }}$ В случае же наличия проводящих сред, благодаря присутствию члена $\sigma \mathbf{E}$, инвариантность уравнений нарушается.

В нашей проблеме мы имеем дело как раз с поглощающими слоями. Желая применить принцип обратимости в этой проблеме, мы должны обобщить его на случай наличия проводящих сред. Искомое обобщение мы получим, очевидно, путем добавления преобразования проводимости при инверсии времени, а именно: $\sigma \rightarrow-\sigma$. Уравнения Максвелла останутся тогда инвариантыми.

Только таким образом, связывая вопрос об обратимости с инвариантностью системы уравнений Максвелла, имеет смысл вводить принцип обратимости. Правда, веществ с отрицательной проводимостью не существует, но этот факт означает лишь то, что электродинамические процессы в проводящих средах (например, распространение света в поглощающем веществе) необратимы. А формально мы имеем право вводить преобразование $\sigma \rightarrow-\sigma$ со всеми вытекающими отсюда следствиямн.

Измене:ию знака проводимости соответствует в оптике переход от данных значений показателей преломления к комплексно-сопряженным значениям. Автор уже ранее $\left.{ }^{5}\right]$ рассматривал многослойные оптические покрытия, у которых показатели преломления слоев (и, возможно, также ограничивающих сред) имеют комплексно-сопряженные значения. Они были названы сопряженными покрытиями, и все величины, относящиеся к ним, обозначались знаком . Такого же обозначения мы будем придерживаться и здесь. 


\section{4. Основные формуль}

Применим теперь метод Книттла [6] к выводу соотношений между амплитудными коэффициентами отражения $\mathbf{r}_{R}, \mathbf{r}_{L}$ и пропускания $\mathbf{t}_{R}$, $t_{L}$ на любом покрытии. Вместо формул, выведенных Книттлом для частного случая непоглошающего покрытия, получим тогда следующие общие формулы (см. $\left.\left.{ }^{7}\right]\right)$ :

$$
\begin{aligned}
& \mathbf{r}_{R} \tilde{\mathbf{r}}_{R}^{*}+\mathbf{t}_{R} \tilde{\mathbf{t}}_{L}^{*}=1 \\
& \mathbf{r}_{R} \tilde{\mathbf{t}}_{R}^{*}+\mathbf{t}_{R} \tilde{\mathbf{r}}_{L}^{*}=0
\end{aligned}
$$

откуда вытекает, что

$$
\mathbf{r}_{L}=-\frac{\tilde{\mathbf{r}}_{R}^{*} \mathbf{t}_{R}}{\tilde{\mathbf{t}}_{R}^{*}}
$$

H

$$
\mathbf{t}_{L}=\frac{1-\mathbf{r}_{R} \tilde{\mathbf{r}}_{R}}{\tilde{\mathbf{t}}_{R}} .
$$

Эти формулы в несколько иной записи были выведены другим путем в цитированной выше работе [5]. А аналогичные формулы (17), (18), (20) и (21) Вашичека ['] верны только для непоглощаюших покрытий.

\section{5. Формула Мурмана}

Применим формулы (3) и (4) к вычислению коэффициента отражения света на тонком металлическом слое, используя формулу (1) из статьи Вашичека [ $\left.{ }^{1}\right]$. Подстановка дает:

$$
\mathrm{r}_{R}=\frac{\mathrm{r}_{R}^{\prime}+\frac{\mathrm{r}_{R}^{\prime \prime} \mathbf{t}_{R}^{\prime}}{\tilde{\mathrm{t}}_{R}^{\prime \prime \prime}} e^{-i \mathbf{x}}}{1+\mathrm{r}_{k}^{\prime \prime} \frac{\tilde{\mathbf{r}}_{R}^{\prime \prime} \mathrm{t}_{R}^{\prime}}{\frac{\tilde{t}_{R}^{\prime \prime}}{\mathrm{t}^{\prime}}} e^{-i \mathbf{x}}} .
$$

Но так как $\mathrm{t}_{R}^{\prime}=\frac{2 n_{0}}{\mathbf{n}_{1}+n_{0}}$, то $\tilde{\mathrm{t}}_{R}^{\prime \prime}=\mathrm{t}_{R}^{\prime} \quad$ и формула (5) превращается в формулу Мурмана:

где

$$
\mathrm{r}_{R}=\frac{\mathrm{r}_{R}^{\prime}+\mathrm{r}_{R}^{\prime \prime} e^{-i \mathrm{x}}}{1+\mathrm{r}_{R}^{\prime} \mathrm{r}_{R}^{\prime \prime} e^{-i \mathbf{x}}},
$$

$$
\mathrm{r}_{R}^{\prime \prime}=-\tilde{r}_{L}^{\prime \prime \prime}=-\frac{\mathrm{n}_{1}-n_{2}}{\mathrm{n}_{1}+n_{2}} \text {. }
$$

Таким образом, формула Мурмана получается не с помощью принципа обратимости Власова, как полагает Вашичек, а с помощью принципа обратимости, обобщенного на-случай наличия поглощающнх сред.

Отметим также, что и формулу Мурмана Вашичек применяес неправильно, нменно в примере покрытия, состоящего из слоя диэлектрика и слоя металла. Формула (27) статьи Вашичека ['], вопреки его мненню, не согласуется с формулой Мурмана. Правильная формула такова: 


$$
\mathbf{r}=\frac{r_{1}+r^{\prime \prime \prime} \frac{t_{1}}{\tilde{t}_{1}^{*}} e^{-i x_{2}}}{1+r_{1} r^{\prime \prime \prime} \frac{t_{1}}{\tilde{t}_{1}^{*}} e^{-i x_{2}}},
$$

где $\mathbf{r}_{1}$ дано в формуле (25) статьи Вашичека ['], а $\mathbf{t}_{1}$ есть соотвётствуюший кюэффишиент пропускания:

$$
\mathbf{t}_{1}=\frac{t^{\prime} \mathbf{t}^{\prime \prime} e^{-\frac{i x_{1}}{2}}}{1+r^{\prime} \mathbf{r}^{\prime \prime} e^{-i \mathrm{x}_{1}}}
$$

Формула (8), кстати сказать, основана не столь на формуле Мурмана, сколь на общей формуле (см. формулу (1) в статье [']), с учетом наших формул (1)-(4). Но она, конечно, согласуется и с формулой Мурмана.

\section{6. Заключительные замечания}

Мы должны остановиться здесь еще на некоторых утверждениях Вашичека, которые содержатся в последней части его статьи. Во-первых, Вашичек говорит: «Обе формулы (т. е. формула Мурмана и формула Вашичека. - П. К.) приводят к различным численным результатам, и лишь подробный анализ последних может решить, какая из формул верна». Это утверждение вводит совсем новую точку зрения. До снх пор вопрос решался чисто теоретически, и, по мнению автора настоящей статьи, выбор правильной формулы возможен на этом пути без привлечения численных данных. Правильной является просто та формула, которая вытекает из уравнений Максвелла и из подразумевающегося предположения, что металлический слой является однородным и изотропным и полностью характеризуется своим комплексным показателем преломления. Конечно, экспериментальные численные данные могут расходиться с теоретическими, но причину этого следует искать (как это обычно и делается) в том, что предположение об оптнческой однородности и изотропности слоя является неточным. Реальные слои имеют более сложную структуру, но в нашем спорном вопросе речь идет не о таких слоях. Поэтому ссылка Вашичека (пункт 4-й его выводов) на зависимость структуры слоя от толщины не относитея к вопросу.

Во-вторых, неправильно связывать обсуждаемые формулы с тем, является ли слой сильно или слабо поглощающим. Основная формула (см. формулу (1) в статье Вашичека [']) выводится обычно, правда, нсходя из наглядной картины многократно отражающихся в слое лучей, но, как уже подчеркивалось $\left[{ }^{2}\right]$, она от этого вывода не зависит и єе можно вывести непосредственно на основе граничных условий для векторов поля. Ничего общего не может иметь и принцип обратимости с тем, поглощает ли слой свет сильно или слабо. Странно звучит поэтому утверждение Вашичека о том, что его формула (24) верна только для слабо поглощающих слоев. На самом же деле, если только слой однороден и изотропен, единственно верна в данной проблеме формула Мурмана, и притом независимо от того, имеет ли слой сильную или слабую поглощающую способность.

В заключение автор считает своим долгом выразить уверенность в том, что вызванная воззрениями А. Вашичека научная дискуссия сыграла большую положительную роль в уяснении ряда тонких вопросов теории тонкослойных оптических покрытий. 


\title{
ЛИТЕРАТУРА
}

1. А. В а ш ичек, $\mathrm{K}$ теории отражения света тонким металлическим слоем, Изв. АН Эст. ССР, Серия физ.-мат. и техн. наук, т. IX, o 3, 1960, 242.

2. П. Г. К а рд, К теории отражения и пропускания света тонким металлическим слоем, Изв. АН Эст. ССР, Серия физ.-мат. и техн. наук, т. VII, № 4, 1958, 283.

3. H. M th rmann. Die optischen Konstanten durchsichtigen Silbers, Zs. f. Phys., 80, $1933,161$.

4. А. А. В л а сов, Макроскопическая электродинамика, М., 1955, 40.

5. П. Г. К а рд, Новые рекуррентные формулы в теории многослойных оптических покрытий, Оптика и спектроскопия, 9, 1960, 95.

6. Z. Knittl, The Use of the Principle of Reversibility in Deriving Known Relations for a System of Optical Thin Films, Czechos!. Journ. Phys., 7, 1957, 427.

7. П. Г. К а рд, Оптический принцип обратимости и его применение в теорин тонкослойных покрытий, Оптнка и спектроскопия, 11, 1961, 237.

Тартускиї

государственный университет
Піоступила в редакцию 31. VIII 1960.

\section{VEEL KORD A. VASICEEK'I VALEMITEST}

\author{
P. Kard, \\ Eesti NSV Teaduste Akadeemia korrespondeeriv liige
}

\section{Resümee}

Autori kritiseerivale artiklile $\left[{ }^{2}\right]$ vastuseks kirjutatud artiklis [ $\left.{ }^{1}\right]$ püüab Vašiček pōhjendada oma teooriat uuel viisil, nimelt pööratavuse printsiibi abil. Seejuures aga jäävad kriitikas esiletoodud väited kummutamata, mistōttu püsib ka vastuolu Maxwell’i teooria ja Vašiček’i valemite vahel. Samuti ei pea endiselt paika Vašiček’i väide, et valgust neelavate optiliste katete teooria oma senisel kujul on vastuolus energia jäävuse seadusega. Teiselt poolt näidatakse artiklis, et Vašiček rakendab pööratavuse printsiipi ebaõigesti, mistōttu ka tema järeldused on ekslikud. Näidatakse, kuidas tuleb õigesti rakendada pööratavuse printsiipi. Antud formuleeringust tuletatakse Knittl'i meetodil [ ${ }^{6}$ ] just need valemid, mida Vašiček püüab revideerida. Uhtlasi saadakse mitmekihiliste neelavate katete peegeldumise ja läbilaskvuse koefitsientide vahelised pōhiseosed, mis autor on juba varem ka teisel viisil tuletanud [5].

Tartu Riiklik Olikool

Saabus toimetusse

31. VIII 1960

\section{NOCHMALS UBER A. VASICEK'S FORMELN}

\section{P. Kard}

\section{Zusammenjassung}

In seiner auf den kritischen Aufsatz $\left[{ }^{2}\right]$ des Verfassers geschriebenen Antwort [ $\left.{ }^{1}\right]$ ist Vašiček bestrebt, seine Theorie in neuer Weise, nämlich mit Hilfe des Reversibilitätsprinzips zu begründen. Dabei bleiben aber die im $\left[{ }^{2}\right]$ dargelegten Ausführungen unwiderlegt. Dadurch ist auch der Widerspruch zwischen Maxwell's Theorie und Vašiček's Formcln stehengeblieben. Ebenso erweist sich als haltlos die Behauptung Vašiček's, dass die bisherige Theorie der lichtabsorbierenden optischen Schichtsysteme dem Gesetze der Erhaltung der Energie widerspreche. Andererseits wird es gezeigt, dass Vašiček das Reversibilitätsprinzip in unrichtiger Weise angewandt hat, weshalb auch seine Resultate irrtümlich sind. Es wird gezeigt, wie man das Reversibilitätsprinzip in richtiger Weise anzuwenden hat. Aus der gegebenen Fassung erhält man dann nach dem Verfahren Knittl's [] genau dieselben Formeln, die Vašiček zu revidieren sucht. Zugleich erhält man die Grundbeziehungen zwischen den Reflexions- und Transmissionskoeffizienten der absorbierenden Mehrschichtsysteme, die vom Autor schon früher $\left.{ }^{[5}\right]$ in anderer Weise abgeleitet worden sind.

\section{Staatsuniversität} zu Tartu
Eingegangen am 31. Aug. 1960 